\title{
Building a model of an intelligent agent activity modes
}

\author{
Gennady P. Vinogradov \\ Tver state technical University \\ Tver, Russia \\ wgp272ng@mail.ru
}

\author{
Natalia G. Vinogradova \\ Tver state technical University \\ Tver, Russia \\ n-natalya@mail.ru
}

\begin{abstract}
The paper discusses the problem of simulating successful agent experience for the purpose of its transmission and development. The approach is based on the advanced method of search in the state-space. It is suggested to develop the model on the basis on the four simulation positions with the subsequent integration of the results obtained.
\end{abstract}

Keywords - expert environments, simulation, decisionmaking, science-intensive production.

\section{INTRODUCTION}

The model of choosing an intelligent agent with the subjectively rational form of behavior is given in $[1,2,5$, 6-9]. It is demonstrated that its activity is regulated by cognitive models of purposeful actions. They are implemented in the courses of action [3]. Through the courses of action the agent purposefully influences objects of its environment. The basis for the courses of action development should be found in the agent's subjective ideas of the purposeful state situation [4]. We will assume that the agent's activity observed is the function of the programs that it has worked out, these in their turn being the result of the agent's inherent cognitive processes. Thus, the activity plan and its implementation are the internal programming product. Its result is an action program (algorithm) that uses the operations open to the agent. Its implementation allows the agent to convert the observed situation into the desired one. Successful experience transmission and development make urgent the problem of simulating this experience by analyzing the agent's language patterns and nonverbal communication. The objectives of the activity simulation may be the following:

1. To understand the subject area better by deepening "metaknowledge" underlying its processes in order to teach others or to use new knowledge as an aid.

2. To improve the results of a certain agent's actions by integrating new operations which are used by experienced specialists and contribute to maximum success.

3. To integrate the experience in order to achieve the best results. In these cases, the model of the activity of several agents showing the best results is created.

4. To use activity processes being simulated to create algorithms of courses of action in other domains.

\section{APPROACH TO AgENT's COURSES OF ACTION SIMULATION}

Behavior simulation will be based on the assumption that the agent has a certain set of elementary operations, using which it constructs complex actions applicable to a particular domain, and that on the basis of the concepts available it can construct a sequence of operations. If the agent does not doubt the possibility of implementing each elementary operation, the algorithm comprehension makes the agent sure of the algorithm performability. Thus, the algorithmic description of the course of actions should be regarded as a search for the possible operation sequence in the state-space. This approach can be formalized by using a graph theory, which allows exploring the effectiveness of the algorithms obtained.

Since an elementary operation is associated with some concept, the identification of the action algorithm is accompanied by the construction of the ontology tree of the concepts that the agent manipulates while working-out the algorithm.

To construct a course of action model the agent's speech patterns and nonverbal reactions are analyzed. The result of this analysis is the step-by-step strategies and techniques, which serve the basis for the algorithm construction. At the same time the ontology tree is analyzed to identify the potential of "upper ontologies". New sets of step-by-step operations based on such ground are themselves a foundation for more effective algorithm construction. The central concept in this approach to simulating the agent's activity is a concept of a tree in the state space. A tree root is the original description of the situation, with the branches being the changes of the initial state possible due to the available operations. Forming the algorithm of a course of action involves setting an initial state and the rules of the tree formation (i. e. possible operations to change the current state). The procedure of a tree creation on the basis of such information is termed a generative procedure. The simulation process discloses heuristics that the agent uses to narrow the space of possible options. Their set is an individual characteristic of the agent. Heuristics in the search procedure are used to describe the estimating function as well as the procedures of work grades formation. Their purpose is to reduce the number of variants of strategies while searching for the algorithmic course of action description. The statistical estimating function is almost entirely dependent on the task, and it encompasses 
the agent's knowledge and experience. The detection of the estimating function the agent uses is one of the central challenges when simulating the process of the course of action construction using the algorithmic approach.

We assume that a certain initial state is estimated with an attribute set. The reactions and courses of action base is formed and leads to certain results. This helps to restore the sequence of operations as well as the type of evaluating function applied in each case. In order to determine the type of this function more quickly the agent's conclusion about the search rules should be used.

The procedures of work grades formation are to clarify the idea about the quality of the new situation of the motivated state. They can be used to determine which child nodes should be disclosed and how their estimating function values are combined.

The algorithms of courses of action are the techniques that represent the sequence of operations leading to task completion. Their effectiveness depends on the abilities and skills that connect beliefs and values with a certain behavior. These abilities and skills provide communication and "levers" to manifest a situation vision and identification, and values and beliefs as actions in a particular environment. They allow the agent to instantly reproduce a particular skill at any time and under the conditions of any problem, situation or context. This means that all mental and behavioral programs are associated with and determined by the purpose, values, norms and the available means of goal achievement. This means that at each stage of the course of action construction, the agent thinks and (consciously and unconsciously) determines the goals and develops a procedure to check whether or not the goal has been reached. If the goal has not been reached, the agent, by means of some changes or in some other way determines the means of approaching the desired goal. When the test criteria are satisfied, the agent moves to the next stage of constructing the course of action. Thus, the function of any part of the behavioral program is to check the incoming information, to evaluate its progress or to undertake some action to alter a certain part of the actual experience so that it could meet the test criteria and it could be possible to move on to the next algorithm portion implementation. As [5] shows, a criterion that the agent uses could be one of the two indices: the specific value of the result, and the specific value of the motivated state effectiveness in a new situation. There are many ways of constructing such indices because they are based on the entire structure of the agent's ideas, which suggests a variety of ideas built on various assumptions and justifications. By varying these justifications, fundamentally different results of the agent's activity can be determined. Errors in justifications and verification methods not caught by the agent, lead to the actions which serve the difference between effective and ineffective performance. Consequently, in order to successfully simulate the result obtained, the following key elements in their relation to the result observed have to be identified:

1. The goals set by the agent.
2. The form of these goals from the agent's perspective.

3. The justifications and justification procedures used by the agent to determine the action effectiveness and success.

4. Cognitive steps that the agent uses to construct the course of action.

5. Actions that the agent undertakes to achieve the goal, as well as the specific types of behavior as a means of these actions implementation.

6. The agent's reaction if the goal is not reached on the first try.

\section{Simulation BASEline}

Simulation suggests the description of courses of action from various positions. There are four basic perception positions, from which the data is collected and interpreted: the first position (a person's own point of view), the second position (looking at the situation from another person's point of view), the third position (regarding the situation from a disinterested observer's point of view), the fourth position (considering the situation from the point of view of a system involved in the situation).

As it is assumed that each position uses different ideas of the situation and possible courses of action, the integration of the points of view and their agreement allows the agent to expand its understanding of the motivated state situation.

Simulating from the first position means to try to do something on one's own and explore the way this is done. The analysis of the course of action is carried out from the researcher's point of view. The second position seems to suggest complete simulation of the agent's behavior, when a researcher tries to think and act as close to the agent's thoughts and actions as possible. This approach allows intuitive understanding of significant but unconscious aspects of the simulated agent's thoughts and actions. To simulate from the third position is to observe the behavior of the simulated agent as a disinterested observer. The third method involves the construction of a course of action model in terms of a specific scientific discipline related to the agent's subject area. The fourth position suggests a kind of intuitive synthesis of all the ideas obtained in order to create a model characterized by maximum figures of the result and efficiency specific values.

This approach suggests the use of implicit and explicit information. The fact is that there may be a situation in which the agent knows or understands the main point of some activity, but is unable to carry it out (conscious incompetence). And vice versa the agent is capable to perform some actions well, but does not understand how it is done (unconscious competence). Being skilled implies both the ability to "do what you know" and the ability to "know what you are doing." Nevertheless, many of the behavioral and psychological elements ensuring agents' successful actions remain largely unconscious and only intuitively understandable to them. As a result, they are unable to directly describe the mechanisms underlying any abilities. Moreover, some agents deliberately avoid thinking about what they do and how they do it, for fear that this 
knowledge will prevent them from acting intuitively. Therefore, one of simulation goals is to reveal and identify unconscious competence and to bring it to mind with the purpose of better skill understanding, improvement and transfer.

Cognitive and behavioral competence can be simulated either "implicitly" or "explicitly". Implicit simulation suggests taking the second position in respect of the simulation subject in order to achieve an intuitive understanding of this person's subjective experience. Explicit simulation means stepping into the third position so as to describe the agent's explicit experience structure so that it can be passed to others. Implicit simulation is primarily an inductive process by which we receive and perceive the structures of the world. Explicit modeling is essentially a deductive process by which we describe and realize our world perception. Both processes are needed for successful simulation. Without the "implicit" phase there can be no effective intuitive base on which to build the "explicit" model. On the other hand, without the "explicit" phase simulated information can be neither embodied in methods and means nor be transmitted to others. Implicit simulation by itself helps a person to develop a personal, unconscious skill in connection with the desired behavior (usually this is the way young children learn). However, the creation of technology, machinery or skills that can be taught, or transmitted to others, in a sense, requires explicit simulation.

The result should be a model which synthesizes (a) an intuitive understanding of the agent's abilities, (b) direct observation of the agent's work, and (c) the researcher's explicit knowledge of the agent's subject area. The main simulation phase reflects the movement from the implicit simulation to the explicit one.

Preparation. It implies the choice of a person with an ability to simulate and the determination of (a) the simulation context; (b) the method of observing the simulated person; (c) preferable relations with the simulated person; (d) the researcher's state in the course of the simulation process.

In addition, this phase includes the creation of the conditions that will allow total plunging into the observation and analysis process.

Phase 1. Intuitive understanding. The first phase of the simulation process includes involving the simulated person into a desired activity or an ability realization in an appropriate context. Simulation starts with taking the "second position" in order to come to an intuitive understanding of the skill which the agent demonstrates. There is no need to attempt determining the behavior structure. It is necessary to enter a model state and to identify oneself with them mentally imitating the agent's action. The agent's external behavior is a surface structure. The second position allows getting information about its deep background structure. This is the phase of the simulated agent's ideas self-consciousness, or the phase of "unconscious understanding." Once an adequate level of intuitive understanding of the studied ability is reached, it is necessary to create a context in which to use this ability, and apply it, acting the way the agent acts. Then an attempt to achieve the same result being "oneself" is to be made. In this way, a "double description" of the simulated skill is obtained. The first simulation phase ends with obtaining approximately the same results that the agent has.

Phase 2. Abstraction. The next stage of the simulation process is the separation of essential elements from random, irrelevant ones. At this stage, the simulated strategies and behavior types become more explicit. As an ability to achieve the results similar to the agent's results is achieved, it is necessary to construct a course of action on the basis of the researcher's ideas in order to achieve the same results by using the "first position."

The task is to identify and define specific cognitive and behavioral steps necessary to achieve the desired result within a chosen context (or contexts). To do this, a method of "bracketing" the elements of the identified strategies or behaviors is used, to evaluate their significance. If, in the absence of some element, the reactions remain unchanged, the element is considered not essential for the model. If a result changes due to the absence of some element, this means that something important has been found. The purpose of this procedure is to bring the simulated actions to the simplest and most elegant forms, as well as to put first things first.

Upon this phase completion there will be received a "minimal model" of understanding the agent's ability from their own point of view (i. e. from the "first position") and developed an intuitive understanding of the agent's ability from the "second position". In addition, there will also be the "third position", a perspective, which will show the difference between the way a simulated ability is reproduced and the way the person shows this ability.

Phase 3. Integration. The final simulation stage involves the construction of the context and procedures that would allow others to learn the skills simulated, and therefore to get the same results as a model person. In order to create a needed plan, it is necessary to synthesize the information received in all the positions of perception. In contrast to simple (incremental) simulation, i. e. the simulated person's activities imitation, the most effective way is the creation of an appropriate reference experience, which allows learners to discover and develop a "sequence of operations" required for successful skill implementation. To acquire this skill it is not necessary to go through the whole simulation process. It should be noted again, that the guideline at this stage is the "utility" of the courses of action for those agents for whom the model is designed.

\section{CONCLUSIONS}

The article discusses the problem of simulating the agent's courses of action and suggests the basic simulation steps arranged in the following sequence:

1. To determine a specialist, whose ability needs to be simulated, and the contexts in which he or she uses this ability. 
2. To collect information in the relevant contexts and from different perceptual positions, starting with an intuitive understanding of the ability from the "second position", and then attempting to reproduce the results from the "first position." Using the "third position", to determine the difference between the courses of action on the basis of explicit information, and the agent's actions.

3. To filter the information collected in order to single out significant cognitive and behavioral patterns.

4. To reduce these patterns to a logically coherent framework or "model."

5. To validate the effectiveness and usefulness of the model constructed by testing it in different contexts and situations so as to be sure that it can be the basis for the desired results.

6. To reduce the model in order to identify the simplest and most elegant forms, allowing the desired results achievement.

7. To frame the information influence on transmission or setting the courses of action identified during a simulation process.

8. To determine the model utility measurement tools and parameters, as well as the field of its application.
The approach suggested in the paper quite logically fits into the methodology of synthesizing technological processes of machining based on the design process decomposition, as shown in [1,2].

\section{REFERENCES}

[1]. Wooldridge M. J. The Logical Modeling of Computational MultiAgent Systems. PhD thesis. Manchester. 1992, 153 p.

[2]. Wooldridge M. J. Intelligent Agents // Multiagent Systems. 2001, pp. 27.79 .

[3]. Tarasov V. B. Ot mnogoagentnyh system $\mathrm{k}$ intellektualnym organizacijam: filosofija, psichologija, informatika / V. B. Tarasov.Moskva: Editorial URS, 2002.

[4]. Vinogradov, G. Decision Making based of Subjective Conceptions of Decision Situation / G. Vinogradov //. Interactive Systems and Technologies: the Problems of Human-Computer Interaction. Collection of Scientific papers. Uljanovsk, 2011, pp. 403-414.

[5]. Vinogradov, G. P. Modelirovanije povedenija agenta $\mathrm{s}$ uchetom subjektivnyh predstavlenij o situatcii vybora/ G. P. Vinogradov, V. N. Kuznetcov// Iskusstvehhyj Intellekt I Priniatie Reshenij. vol. 3, pp. 58-72.

[6]. Wooldridge M. J. Intelligent Agents // Multiagent Systems. 2001. P. 27.79 .

[7]. Wooldridge M. J., Jennings N.R. Intelligent Agents: Theory and Practice // The $\sim$ nowledge Engineering Review. 1995.

[8]. Huhns M.N., Stephens L.M. Multiagent Systems and Societies of Agents // Multiagent Systems. 2001, pp. 79.121.

[9]. Jennings N.R., Wooldridge M. J. Applications of Intelligent Agents._London: ueen Mary $€$ West.eld College, University of London. 2000. 27 p. 